\title{
Pigmented villonodular synovitis of the knee: a case report
}

F.C Sitati, MBChB, MMed(Orth, Makerere), FCS(ECSA), PCEA Kikuyu Hospital, Orthopaedic and rehabilitation Centre, Kenya

\section{SUMMARY}

This case study is a report of a 21 year old female with one year history of non-traumatic left knee pain and swelling. Radiography and histology were in keeping with Pigmented Villonodular Synovitis (PVNS). This case highlights the clinical presentation of this rare disorder and emphasizes its consideration as a differential diagnosis in our set up when dealing with non-traumatic persistent knee pain and swelling.

\section{INTRODUCTION}

A 21 year old female presented in our unit with a one year history of left knee pain and swelling. She reported generalized left knee pain of insidious onset which progressively increased in severity. It was aggravated by activity. She denied any history of trauma, weight loss, anorexia or fevers. Review of the respiratory, cardiovascular, gastrointestinal, genitourinary and nervous systems was essentially normal. Her past medical and family history were unremarkable.

\section{CASE REPORT}

Examination findings: The general physical examination was unremarkable, except for the presence of a left sided antalgic gait. Local examination of the left knee revealed generalized swelling and increased local temperature. The knee had active range of motion of $0^{\circ}-60^{\circ}$. Neurovascular status was normal. Aspiration of the knee revealed reddish brown joint fluid.

Investigations: A plain radiograph of the left knee revealed medial tibial plateau juxtaarticular erosion (Figure 1). No CT scan or MRI were done. Complete blood count was normal.ESR was $25 \mathrm{~mm} / \mathrm{hr}$. Rheumatoid factor, alkaline phosphatase, calcium and phosphate levels were normal. The patient underwent an open biopsy of the left knee through a medial parapatellar approach. The synovium appeared thickened and profuse amounts of grape like tissue were obtained with reddish brown joint fluid. Samples of the synovium, nodular tissue and fluid were taken for both histology and bacteriology. The knee was debrided of the nodular tissue and thoroughly lavaged.
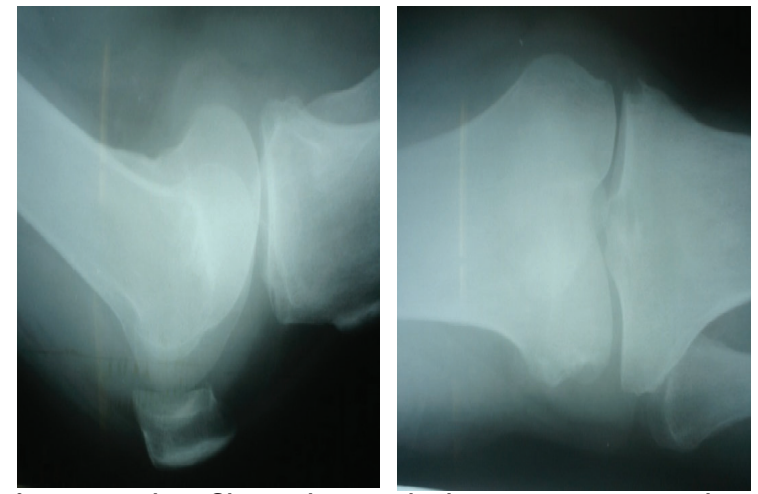

Figure 1: Plain film radiograph showing a cyst involving juxta articular medial tibial plateau.

Histological examination demonstrated villous like processes infiltrated by inflammatory cells mainly lymphocytes and plasma cells and multinucleated giant cells highly suggestive of villonodular synovitis. Microbiology results revealed no micro-organisms on Gram stain or ZN stain and no growth on culture.

The patient was discharged on NSAIDs for one month. The post operative course was uneventful. At one month follow-up she reported marked decrease in the knee pain and swelling and had a knee range of motion of $0^{\circ}-90^{\circ}$. At three months follow-up, the patient had little discomfort in the knee and was not using any medication for her knee.

\section{DISCUSSION}

Pigmented villonodular synovitis (PVNS) is a rare benign proliferative disorder of uncertain etiology that affects synovial lined joints, bursae, and tendon sheaths. The disorder results in various degrees of villous and/or nodular changes in the affected structures. Annual incidence of PVNS in the United states is estimated at 2 cases per million population; incidence of the localized 
form is 9 cases per million (1). In our set up, there is no published literature on this condition, thus the need to highlight this rare case.

The genesis of PVNS is disputed. Most authors regard PVNS as a reactive process (2), a chronic inflammatory process, benign neoplasm(3) or having a genetic component (4).

PVNS has been described in paediatric and elderly populations, but it occurs more often in patients aged 20-50 years. The joint most often affected is the knee (approximately 80\%), with the hip, ankle, and shoulder being less commonly impacted $(5,6)$. The disease usually is monoarticular although a few polyarticular cases have been reported (4). Patients usually present with painless joint swelling of insidious onset that mimics joint effusion. Joint pain subsequently supervenes, but the swelling is disproportionate to the degree of pain. The pain is mild and of insidious onset, and it progressively worsens and frequently is accompanied by decreased range of motion and locking of the joint. Recurrent mild to moderate effusion creates the impression of recurrent joint swelling (5). In our patient, knee pain and swelling together with stiffness were the main features, this is in keeping with Dowarts et al observations (15). The differential diagnosis of PVNS on plain film with multiple subchondral cysts includes DJD, tuberculosis, haemophilia, amyloidosis, gout and synovial chondromatosis (7).

The laboratory investigations were normal as expected. The radiographs reveal typical radiolucent defects usually cystic with a thin sclerotic rim. Cortical erosions and joint space narrowing are more common in the hip than the knee $(8,9)$. Our patient had morbidity associated with the pain and decreased knee motion.

The treatment of this disease is challenging (8). Nonsurgical treatment includes use of triamcinolone hexacetonid, use of ${ }^{90} Y$ ttrium or radiotherapy $(10,11)$. Surgical treatment by synovectomy has appeared to be the most effective in symptomatic diffuse type PVNS (12). Joint replacement is also an option however the low age of these otherwise health patients may result in early failure (8).

\section{CONCLUSION}

PVNS is a rare condition. The clinical presentation of one case found in our region is described. Patients usually present with insidious onset joint swelling associated with pain that mimics joint effusion. Joint pain subsequently supervenes, but the swelling is disproportionate to the degree of pain. The pain is mild and of insidious onset, and it progressively worsens and frequently is accompanied by decreased range of motion and sometimes locking of the joint. We recommend that PVNS should be included as a differential diagnosis when evaluating a young adult with non-traumatic persistent knee pain and swelling.

\section{REFERENCES}

1. DiCaprio, M.R., Damron, T.A., Stadnick, M., et al. Pigmented villonodular synovitis of the elbow: a case report and literature review. J. Hand Surg. (Am). 1999; 24:386-391.

2. Sakkers, R. J., de Jong, D. and van der Heul, R. O. $\mathrm{X}$-chromosome inactivation in patients who have pigmented villonodular synovitis. J. Bone Joint Surg. (Am) 1991; 73: 1532-1536.

3. Choong, P. F., Willen, H., Nilbert, M., et al. Pigmented villonodular synovitis. Monoclonality and metastases - a case for neoplastic origin? Acta. Orthop. Scand. 1995; 66: 64-68.

4. Wendt, R. G., Wolfe, F., McQueen, D., et al. Polyarticular pigmented villonodular synovitis in children:evidence for a genetic contribution. J. Rheumatol. 1986; 13: 921-926.

5. Dorwart, R.H., Genant, H.K., Johnston, W.H., et al. Pigmented villonodular synovitis of synovial joints: clinical, pathologic, and radiologic features. AJR Am. J. Roentgenol. 1984; 143: 877-885.

6. Uschijima, M., Hashimoto, H., Tsuneyoshi, M. and Enjoji, M. Pigmented villonodular synovitis. A clinicopathologic study of 52 cases. Acta. Pathol. Jpn. 1986; 36: 317-326.

7. Goldman, A.B. and DiCarlo, E.F. Pigmented villonodular synovitis. Diagnosis and differential diagnosis. Radiol. Clin. North Am. 1988; 26:1327-1347.

8. Rydholm, U. Pigmented villonodular synovitis of the hip joint. Int. Orthop. 1987; 11: 307-310.

9. Cotton, A., Flipo, R. M., Chastanet, P., et al. Pigmented villonodular synovitis of the hip joint: review of radiographic features in 58 patients. Skeletal Radiol. 1995; 24: 1-6.

10. Wiss, D. A. Recurrent villonodular synovitis of the knee. Successful treatment with Yttrium-90. Clin. Orthop. 1982; 169: 140-144.

11. O'Sullivan, B., Cummings, B., Catton, C., et al. Outcome following radiation treatment for high-risk pigmented villonodular synovitis. Int. J. Radiat. Oncol. Biol. Phys. 1995; 32: 777-786.

12. Sim, F. H. Synovial proliferative disorders: role of synovectomy. Arthroscopy. 1985; 1: 198-204. 